\title{
Infiltrating Bladder Urothelial Carcinoma, Microcystic Variant
}

National Cancer Institute

\section{Source}

National Cancer Institute. Infiltrating Bladder Urothelial Carcinoma, Microcystic Variant. NCl Thesaurus. Code C39820.

A variant of infiltrating bladder urothelial carcinoma. It is characterized by microcysts formation. It is also associated with intracytoplasmic mucin deposits and calcification in cyst walls. 